\title{
Méthode de sélection de lacs de référence dans le cadre d'une étude Before-After Control-Impact (BACI) évaluant les effets des coupes forestières sur le zooplancton des lacs de la forêt boréale Selection method for reference lakes in a Before-After
Control-Impact (BACI) design study assessing the impacts of
forest harvesting on zooplankton in boreal lakes
}

\author{
L. Duhaime et B. Pinel-Alloul
}

Volume 18, numéro hors-série, 2005

URI : https://id.erudit.org/iderudit/705584ar

DOI : https://doi.org/10.7202/705584ar

Aller au sommaire du numéro

Éditeur(s)

Université du Québec - INRS-Eau, Terre et Environnement (INRS-ETE)

ISSN

0992-7158 (imprimé)

1718-8598 (numérique)

Découvrir la revue

Citer cet article

Duhaime, L. \& Pinel-Alloul, B. (2005). Méthode de sélection de lacs de référence dans le cadre d'une étude Before-After Control-Impact (BACI) évaluant les effets des coupes forestières sur le zooplancton des lacs de la forêt boréale. Revue des sciences de l'eau / Journal of Water Science, 18, 199-220.

https://doi.org/10.7202/705584ar

\section{Résumé de l'article}

Plusieurs approches tentent de mesurer l'impact des perturbations anthropiques sur les écosystèmes. L'approche BACI (Before-After Control-Impact) consiste à suivre deux groupes de sites (contrôle et impact), avant et après une perturbation, afin de mesurer l'effet de cette dernière sur les écosystèmes. Les études BACI permettent de contrôler la variabilité naturelle entre les groupes de sites, par le suivi des mêmes sites d'impact avant et après la perturbation, tout en minimisant la variabilité naturelle entre les années grâce au suivi de sites de contrôle échantillonnés également avant et après la perturbation. Puisque la variation naturelle entre les années dans les sites d'impact est estimée à partir de celle des sites de contrôle, il est nécessaire de sélectionner des sites de contrôle dont les caractéristiques limnologiques sont semblables à celles des sites d'impact. Ceci est essentiel pour une bonne application de l'approche BACI, afin de s'assurer que les sites naturels et perturbés répondent de la même façon aux variations naturelles interannuelles dans l'environnement et que les différences observées dans les sites d'impact avant et après la perturbation soient attribuables à celle-ci.

Cet article propose une méthode de sélection des sites de contrôle dans le cadre d'une étude BACI portant sur l'impact des coupes forestières sur le zooplancton des lacs de la forêt boréale au Québec. Le zooplancton de 16 lacs de la forêt boréale a été échantillonné un an avant (2000) et deux ans après (2001-2002) des coupes forestières sur le bassin versant de certains lacs. Six lacs ont subi des coupes importantes sur 44 à $77 \%$ du bassin versant (lacs de coupe: DA2, DF2, DF7, DF9, K4, K8) et 10 lacs sont restés à l'état naturel ou n'ont subi que des coupes négligeables sur moins de $2 \%$ du bassin versant (lacs de référence: K2, AB34, AB35, AB40, CSL5, DA4, DF4, N35, N89, N43). Parmi ces dix lacs de référence, nous avons sélectionné les six lacs les plus semblables aux lacs de coupe, à l'aide d'analyses en composantes principales (ACP) basées sur la similarité des variables morphométriques, de la qualité de l'eau et du zooplancton avant la coupe (2000). De plus, les variables ayant la plus grande contribution à la variation totale au niveau de ces trois groupes de variables ont été déterminées. Quatre lacs ont été exclus (K2, N89, AB35, AB40) et six lakes (AB34, CSL5, DA4, DF4, N35, N43) ont été sélectionnés comme référence. Finalement, la validité du choix des six lacs de référence a été testée par des analyses de redondance (RDA) avec une variable binaire qui permettait de distinguer les lacs de coupe des lacs de référence sélectionnés. Les analyses de redondance ont montré que les variables de la morphométrie, de la qualité de l'eau et du zooplancton des lacs de référence sélectionnés ne différaient pas significativement de celles des lacs de coupe avant la perturbation. En conséquence, les différences observées après la perturbation dans les six lacs de coupe, relativement aux variations naturelles dans les six lacs de référence sélectionnés, devraient être attribuables à l'effet de la coupe forestière. La méthode de sélection développée dans le cadre de cette étude peut être utilisée pour évaluer à l'aide d'une approche BACI les effets de toute perturbation anthropique sur les écosystèmes
Ce document est protégé par la loi sur le droit d'auteur. L’utilisation des services d'Érudit (y compris la reproduction) est assujettie à sa politique d'utilisation que vous pouvez consulter en ligne.

https://apropos.erudit.org/fr/usagers/politique-dutilisation/ 


\title{
Méthode de sélection de lacs de référence dans le cadre d'une étude Before-After Control-Impact (BACI) évaluant les effets des coupes forestières sur le zooplancton des lacs de la forêt boréale
}

\author{
Selection method for reference lakes in a Before-After \\ Control-Impact (BACl) design study assessing \\ the impacts of forest harvesting on zooplankton \\ in boreal lakes
}

\section{DUHAIME ${ }^{1}$, B. PINEL-ALLOUL ${ }^{1 *}$}

Reçu le 15 novembre 2003, accepté le 27 janvier 2005**.

\section{SUMMARY}

Several designs can be used to assess the effects of human perturbations on ecosystems. However, the main difficulty is to isolate natural sources of variation from the variation induced by the perturbation. Several studies have shown that the natural differences between the reference and the impacted sites may influence their responses to the perturbation. In a comparative design comparing the conditions at the reference sites and the impacted sites after the perturbation, it is not possible to control for the natural sources of variation between these two groups of sites that occurred before the perturbation. This natural variation among sites is taken into account in a Before-After design in which the same sites are monitored before and after perturbation, but the natural sources of variation among years cannot be separated from the variation induced by the perturbation. In this study, we used a BACI (Before-After Control-Impact) design to measure the effect of a perturbation on an ecosystem by following two groups of sites (control and impacted) before and after the perturbation. A BACI design is the only one that allows controlling for the natural variability among sites by following the same impacted sites before and after the perturbation. This approach also takes into account the natural variability among years by monitoring concomitantly the control sites before and after the perturbation. Since the natural variability among years at the impacted sites is estimated by the variability measured at the control sites, it is essential to select control sites that are the most similar to the impacted sites with respect to their limnological characteristics. This is a requirement for the good use of the BACI design, to make sure that

1. GRIL, Département de sciences biologiques, Université de Montréal, CP 6128, succ. Centre ville, Montréal, Qué. H3C 3J7, Canada.

* Correspondance : bernadette.pinel-alloul@umontreal.ca ; lyne.duhaime@umontreal.ca

** Les commentaires seront reçus jusqu'au 31 mai 2006. 
control and impacted sites have the same responses towards year-to-year natural variations in their environment and to ensure that the differences observed at the impacted sites before-after the perturbation are caused by it.

This paper proposes a method for the selection of control sites in a BACI design to study the impact of forest harvesting on zooplankton communities in lakes of the boreal forest in Québec. Zooplankton in 16 lakes was sampled one year before (2000) and two years after (2001-2002) forest harvesting in the watersheds of some lakes. Six lakes were impacted by important harvesting on 44 to $77 \%$ of their watershed (cut lakes: DA2, DF2, DF7, DF9, K4, K8) and ten lakes were considered as natural lakes with forest harvesting on less than $2 \%$ of their watershed (reference lakes: K2, AB34, AB35, AB40, CSL5, DA4, DF4, N35, N89, N43). Among these ten natural lakes, we selected the six reference lakes that had limnological features most similar to the cut lakes, based on the morphometry, water quality and zooplankton variables before forest harvesting (2000). We used principal component analyses (PCA) to compare the lakes (10 natural lakes and six lakes to be impacted by watershed harvesting) using ordination biplots. A PCA was done for each group of variables. Lake volume, maximal depth, and area and the slope of the watershed were the variables having the most important contributions to the total variation in morphometry. Positive correlations were found between the slope of the watershed and the maximal depth of the lake and between the lake area and the lake volume. This PCA allowed us to eliminate two reference lakes that had higher values in lake volume and area for one lake (K2) and in lake volume and maximal depth for the other one (AB35), compared to the cut lakes. When comparing water quality variables, total phosphorus (TP), total nitrogen (TN), dissolved organic carbon (DOC), Secchi depth, $\mathrm{Ca}^{2+}$, $\mathrm{Mg}^{2+}$, alkalinity ( $\mathrm{ALK}$ ) and $\mathrm{pH}$ contributed more to the total variation than other variables. Among these variables, DOC, TP and TN were positively correlated together whereas they were negatively correlated to water transparency (Secchi). Two reference lakes (AB35, N89) had higher transparency and lower values in DOC, TP and TN and one reference lake $(\mathrm{AB} 40)$ had lower values in $\mathrm{pH}, \mathrm{ALK}, \mathrm{Ca}^{2+}$ and $\mathrm{Mg}^{2+}$ compared to cut lakes. Since one of these three lakes had previously been eliminated on the basis of its morphometric variables, we had a total of four reference lakes excluded from the study. The PCA on zooplankton variables revealed that only the largest class of zooplankton ( $>500 \mu \mathrm{m}$ ) contributed significantly to zooplankton total variation. In general, reference lakes and cut lakes had a similar distribution on the ordination biplot so no lake was eliminated with respect to zooplankton variables. Finally, four reference lakes were excluded (K2, N89, AB35, AB40) and six reference lakes were selected (AB34, CSL5, DA4, DF4, N35, N43).

The validation of the selection of the six reference lakes was made using redundancy analyses (RDA) with a binary variable discriminating between the six cut lakes and the six reference lakes selected in the PCAs. Multivariate methods such as RDA have the advantage of testing if the two groups of lakes are different with respect to many variables and not just one, as would be the case when using a univariate method. Redundancy analyses showed that the morphometric, water quality and zooplankton variables in the six selected reference lakes were not significantly different from those in the six cut lakes before harvesting. The RDA results supported the selection of the six reference lakes based on the ordination biplots from the three PCA made on the three groups of variables. We could then presume that any difference observed after the perturbation in the cut lakes, relative to the natural variation among years in the six reference lakes, could be attributed to the effect of forest harvesting. Finally, this method of selection of control sites could be used for any BACI study testing the effects of human perturbation on ecosystems.

Key words: method, selection, reference lakes, BACI design, harvesting, zooplankton, boreal forest. 
Plusieurs approches tentent de mesurer l'impact des perturbations anthropiques sur les écosystèmes. L’approche BACI (Before-After Control-Impact) consiste à suivre deux groupes de sites (contrôle et impact), avant et après une perturbation, afin de mesurer l'effet de cette dernière sur les écosystèmes. Les études BACI permettent de contrôler la variabilité naturelle entre les groupes de sites, par le suivi des mêmes sites d'impact avant et après la perturbation, tout en minimisant la variabilité naturelle entre les années grâce au suivi de sites de contrôle échantillonnés également avant et après la perturbation. Puisque la variation naturelle entre les années dans les sites d'impact est estimée à partir de celle des sites de contrôle, il est nécessaire de sélectionner des sites de contrôle dont les caractéristiques limnologiques sont semblables à celles des sites d'impact. Ceci est essentiel pour une bonne application de l'approche BACI, afin de s'assurer que les sites naturels et perturbés répondent de la même façon aux variations naturelles interannuelles dans l'environnement et que les différences observées dans les sites d'impact avant et après la perturbation soient attribuables à celle-ci.

Cet article propose une méthode de sélection des sites de contrôle dans le cadre d'une étude BACI portant sur l'impact des coupes forestières sur le zooplancton des lacs de la forêt boréale au Québec. Le zooplancton de 16 lacs de la forêt boréale a été échantillonné un an avant (2000) et deux ans après (2001-2002) des coupes forestières sur le bassin versant de certains lacs. Six lacs ont subi des coupes importantes sur 44 à $77 \%$ du bassin versant (lacs de coupe : DA2, DF2, DF7, DF9, K4, K8) et 10 lacs sont restés à l'état naturel ou n'ont subi que des coupes négligeables sur moins de $2 \%$ du bassin versant (lacs de référence : K2, AB34, AB35, AB40, CSL5, DA4, DF4, N35, N89, N43). Parmi ces dix lacs de référence, nous avons sélectionné les six lacs les plus semblables aux lacs de coupe, à l'aide d'analyses en composantes principales (ACP) basées sur la similarité des variables morphométriques, de la qualité de l'eau et du zooplancton avant la coupe (2000). De plus, les variables ayant la plus grande contribution à la variation totale au niveau de ces trois groupes de variables ont été déterminées. Quatre lacs ont été exclus (K2, N89, AB35, AB40) et six lacs (AB34, CSL5, DA4, DF4, N35, N43) ont été sélectionnés comme référence. Finalement, la validité du choix des six lacs de référence a été testée par des analyses de redondance (RDA) avec une variable binaire qui permettait de distinguer les lacs de coupe des lacs de référence sélectionnés. Les analyses de redondance ont montré que les variables de la morphométrie, de la qualité de l'eau et du zooplancton des lacs de référence sélectionnés ne différaient pas significativement de celles des lacs de coupe avant la perturbation. En conséquence, les différences observées après la perturbation dans les six lacs de coupe, relativement aux variations naturelles dans les six lacs de référence sélectionnés, devraient être attribuables à l'effet de la coupe forestière. La méthode de sélection développée dans le cadre de cette étude peut être utilisée pour évaluer à l'aide d'une approche BACI les effets de toute perturbation anthropique sur les écosystèmes.

Mots clés : méthode, sélection, lacs de référence, approche BACI, coupe forestière, zooplancton, forêt boréale. 


\section{1 - INTRODUCTION}

Au cours des dernières décennies, différentes approches ont été mises en avant afin de mesurer l'impact des perturbations anthropiques sur les écosystèmes (GREEN, 1979 ; STEWART-OATEN et al., 1986 ; OSENBERG et SCHMITT, 1996). Dans le cadre d'études évaluant l'effet des coupes forestières sur les écosystèmes lacustres, trois approches principales ont été utilisées : l'approche comparative (Control-Impact Design), l'approche "Before-After Design » et l'approche BACl (Before-After Control-Impact Design).

L'approche comparative (Control-Impact Design) permet de comparer des écosystèmes perturbés à des écosystèmes de référence situés dans la même région, mais assez loin pour ne pas subir les effets de la perturbation (OSENBERG et SCHMITT, 1996). Cette approche suppose que les deux groupes de sites sont identiques en absence de perturbation, prémisse pour le moins irréaliste puisque les écosystèmes présentent une variation naturelle entre les sites. Avec une telle approche, il est impossible d'isoler la variabilité naturelle entre les groupes de lacs de celle causée par la perturbation (figure 1).

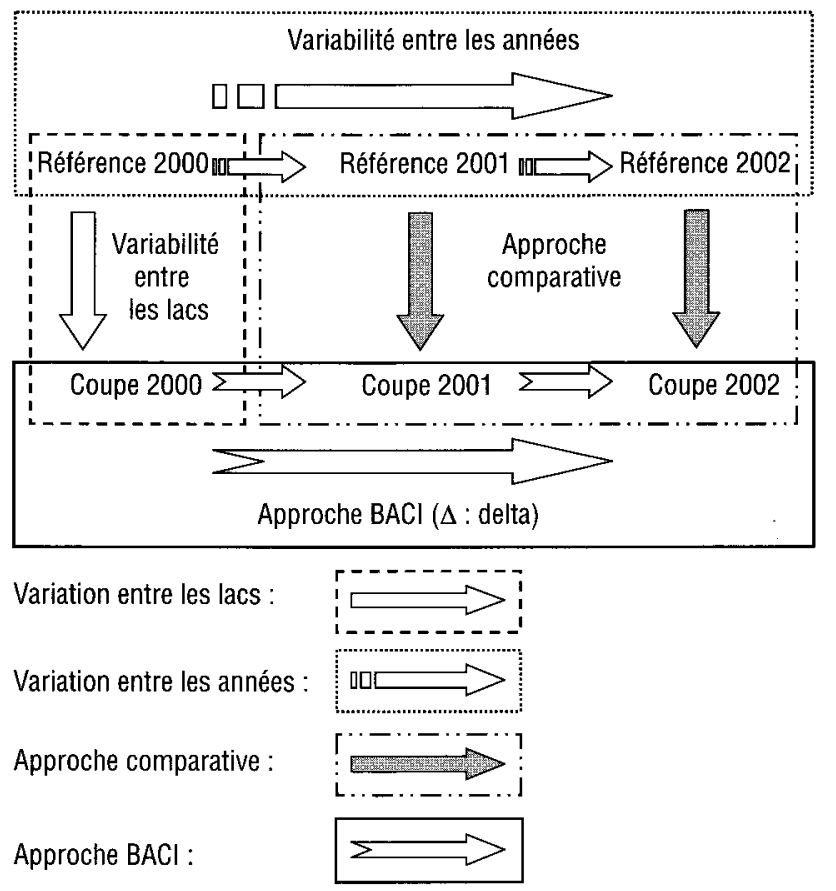

Figure 1 Évaluation des effets des coupes forestières selon l'approche BACl et l'approche comparative et sources de variations naturelles entre les lacs et entre les années. Modifié de Bondallaz (2002).

Assessment of the impacts of havesting following a $\mathrm{BACl}$ design and a comparative design, showing natural sources of variation among lakes and among years. Modified from Bondallaz (2002). 
Pour éliminer cette variabilité naturelle entre les sites, on a proposé l'approche "Before-After Design ", ou « Intervention Analysis " (BOX et TIAO, 1975), qui consiste à échantillonner un ou plusieurs sites avant et après une perturbation (OSENBERG et SCHMITT, 1996). On réduit ainsi la variabilité naturelle entre les sites en suivant les mêmes sites avant et après la perturbation mais cette approche ne permet pas de distinguer l'effet de la perturbation de la variabilité naturelle entre les années pouvant être causée, par exemple, par des changements dans les conditions climatiques avant et après la perturbation.

L'approche BACI (Before-After Control-Impact), quant à elle, tente de combiner les avantages des deux méthodes précédentes (figure 1). Différentes variantes à cette approche ont été proposées. L'approche $\mathrm{BACl}$ originale, décrite par GREEN (1979), consiste à échantillonner un site de contrôle et un site d'impact une fois avant et une fois après la perturbation. Ceci permet de réduire la variabilité naturelle entre les sites en suivant le même site avant et après impact, tout en évaluant la variabilité naturelle entre les années dans un site de contrôle. Toutefois, avec seulement un site d'impact et un site de contrôle, il est difficile de départager l'effet de la perturbation de celui pouvant être attribuable à une variable naturelle modifiant un site et pas l'autre, comme des conditions climatiques particulières à l'un des deux sites. L'approche BACI (Before-After Control-Impact) plus récente décrite par BERNSTEIN et ZALINSKI (1983) et STEWART-OATEN et al. (1986) permet de tenir compte des sources de variations naturelles et de minimiser leur influence. Elle détermine les différences observées dans les variables à l'étude en soustrayant les valeurs mesurées dans plusieurs sites de contrôle de celles mesurées dans des sites d'impact, avant et après une perturbation. L'échantillonnage peut avoir lieu une ou plusieurs fois par année, mais en même temps pour les sites de contrôle et d'impact, avant et après la perturbation. À chaque date d'échantillonnage, on calcule la différence dans les variables écologiques entre la moyenne de tous les sites de contrôle et la valeur de chacun des sites d'impact ( $\Delta$ : delta) (figure 1). On teste ensuite s'il y a un changement significatif entre ces différences $(\Delta)$ avant et après la perturbation. Pour les variables à l'étude, nous pouvons donc mesurer leur variation avant et après la perturbation dans les sites d'impacts (variation naturelle entre les années et variation due à la perturbation) comparativement aux sites de contrôle (variation naturelle entre les années seulement). Le suivi des mêmes sites d'impact avant et après la perturbation réduit l'effet de la variation naturelle entre les sites, le suivi de plusieurs sites perturbés minimise l'effet d'une variation naturelle isolée à un site précis tandis que le suivi de plusieurs sites de contrôle permet de contrôler pour la variabilité naturelle entre les années avant et après la perturbation. Cette approche est donc celle permettant le mieux d'isoler la variation attribuable à la perturbation de la variation naturelle entre les sites d'impact et de contrôle, et de la variation naturelle entre les années au cours du suivi avant et après la perturbation (STEWART-OATEN et BENCE, 2001).

La plupart des récentes études sur l'effet des coupes forestières sur les écosystèmes lacustres ont adopté une approche comparative (CARIGNAN et al., 2000). De telles études ne considèrent pas la variabilité naturelle entre les groupes de lacs (lacs de coupe vs lacs de référence) et assument que les sites perturbés et les sites de contrôle sont similaires avant la perturbation. Par ailleurs, il a été démontré que la variabilité naturelle entre les lacs de coupe et les lacs de référence peut être importante et qu'elle peut influencer les 
réponses des écosystèmes à la perturbation. II est alors difficile d'isoler l'effet des coupes forestières sur la qualité de l'eau (LEEHMANN, 1994) ou sur la biomasse et la structure en taille des communautés de zooplancton (PATOINE et al., 2000, 2002a, 2002b) des sources de variations naturelles.

Quelques études ont utilisé le design «Before-After Impact 》 (STEEDMAN, 2000 ; STEEDMAN et KUSHNERIUK, 2000; PREPAS et al., 2001) en suivant plusieurs lacs avant et après des coupes sur leur bassin versant, mais sans estimer la variation naturelle par le suivi de lacs de référence. Avec ce design, il est difficile de séparer la variation causée par les coupes forestières de la variabilité naturelle entre les années causée par les changements du climat d'une année à l'autre. Les variations climatiques entre les années peuvent, entre autres, modifier les apports en nutriments et en carbone organique dissous (COD) dans les lacs, éléments susceptibles d'apporter des changements dans la communauté zooplanctonique. Ainsi, PREPAS et al. (2001) ont noté, dans les lacs de la plaine boréale, de plus grandes concentrations en phosphore total (PT) après des coupes forestières seulement les années de fortes pluies à cause du ruissellement plus important sur le bassin versant. II est alors difficile de statuer si cette augmentation de PT est due à la perturbation ou à une forte pluviosité. SCHINDLER et al. (1996) rapportent que l'exportation de COD dans les bassins versants, de même que sa concentration dans les lacs du NordOuest de l'Ontario, diminue lorsque la température et les précipitations sont sous la normale. CARIGNAN et al. (2000) mentionnent aussi que les apports en COD et le coefficient d'extinction de la lumière, dans les lacs de la forêt boréale de l'Est du Canada ayant subi une coupe importante sur leur bassin versant, augmentent lors des années de plus grande pluviosité. En conséquence, si les coupes sont faites lors d'une année de sécheresse, on pourrait observer peu ou pas d'effet sur l'exportation de PT et de COD, tandis que cet effet serait très important si les coupes avaient lieu une année de forte pluviosité.

Finalement, l'approche BACl, telle que décrite par BERNSTEIN et ZALINSKI (1983) et STEWART-OATEN et al. (1986), qui tient compte de la variation naturelle dans les sites de référence, n'a été appliquée qu'une seule fois en Finlande par RASK et al. (1998) pour étudier les effets des coupes forestières sur quatre lacs de coupe et un seul lac de référence. Le fait de n'utiliser qu'un seul lac de référence dans cette étude peut s'avérer problématique si ce lac subit, par exemple, une forte précipitation et que les lacs de coupe ne sont pas affectés par cet événement climatique. Rappelons aussi qu'en plus des approches utilisées pour suivre l'effet de perturbations d'origine humaine sur les écosystèmes lacustres, l'habilité à détecter des impacts dépend aussi de l'importance de la perturbation et d'autres facteurs environnementaux liés à la physiographie du bassin versant (CARIGNAN et al., 2000).

L'objectif final de notre recherche est d'évaluer l'effet des coupes forestières sur le zooplancton des lacs de la forêt boréale du Québec, en appliquant pour la première fois l'approche $\mathrm{BACl}$, mais également l'approche comparative, sur un grand nombre de lacs, soit six lacs de référence et six lacs de coupe (figure 1). Toutefois, afin de satisfaire à la prémisse que les caractéristiques des lacs de référence sont similaires à celles des lacs perturbés, pour que la variabilité naturelle entre les années des lacs de référence représente bien celle des lacs de coupe, il est essentiel de faire une sélection parmi les lacs naturels. Dans ce but, nous présentons dans cet article une méthode de sélection permettant de choisir, dans une population de lacs, les lacs de réfé- 
rence les plus semblables aux lacs perturbés et de valider ce choix. La similarité recherchée concerne à la fois les variables morphométriques, de la qualité de l'eau et du zooplancton. Comme l'approche BACl permet de soustraire la variabilité naturelle moyenne du zooplancton des lacs de référence à la variance totale du zooplancton dans chacun des lacs de coupe, les différences significatives de valeurs deltas $(\Delta)$ entre les années avant et après la perturbation, observées dans le zooplancton de chaque lac de coupe, pourront être attribuées essentiellement aux coupes forestières. De plus, la comparaison de deux groupes de lacs semblables avant la perturbation, permettra d'amoindrir la variabilité naturelle entre les sites perturbés et de référence, ce qui donnera plus de validité à l'approche comparative.

\section{2 - MATÉRIEL ET MÉTHODES}

\subsection{Sites d'étude}

Les 16 lacs à l'étude sont situés dans la forêt boréale du bouclier canadien au Québec $\left(47^{\circ} 52^{\prime}-48^{\circ} 60^{\prime} \mathrm{N}, 73^{\circ} 19^{\prime}-76^{\circ} 43^{\prime} \mathrm{O}\right)$ (figure 2). À l'été 2000 , les bassins versants de tous les lacs étaient à l'état naturel. Les coupes forestières ont débuté à l'automne 2000 et se sont poursuivies jusqu'au printemps 2002. Un total de 6 lacs ont été perturbés par des coupes sur 44 à $77 \%$ de leur bassin versant (DA2, DF2, DF7, DF9, K4, K8 : désignés comme lacs de coupe). Les dix autres lacs (K2, AB34, AB35, AB40, CSL5, DA4, DF4, N35, N89 et N43 : désignés comme lacs de référence) avaient leur bassin versant à l'état naturel ou avec moins de $2 \%$ de surface coupée. Parmi ces lacs, nous avons sélectionné les six lacs ayant une morphométrie, une qualité d'eau et une communauté zooplanctonique ressemblant le plus à celles des six lacs de coupe à l'étude. Les méthodes utilisées pour déterminer la morphométrie des lacs et de leur bassin versant et pour analyser la qualité de l'eau ont été décrites par CARIGNAN et al. (2000).

\subsection{Zooplancton}

Les lacs ont été visités deux fois par année (juin et août) en 2000, 2001 et 2002. À chaque visite, trois échantillons de zooplancton ont été récoltés dans la zone profonde de chacun des lacs avec un filet à contre-levier de $53 \mu \mathrm{m}$ d'ouverture de mailles (FILION et al., 1993) sur toute la colonne d'eau (moins d'un mètre au-dessus des sédiments jusqu'à la surface) et dans les zones euphotique ( $>1 \%$ de lumière transmise) et aphotique $(<1 \%$ de lumière transmise). Le zooplancton a été anesthésié avec de l'eau de Seltz (soda) puis conservé dans du formol (4\%). Au laboratoire, chaque échantillon de zooplancton a été ramené à un volume standard de $200 \mathrm{ml}$ après avoir enlevé les prédateurs invertébrés tels que Chaoborus, Leptodora et autres organismes (larves d'insectes) exclus de l'analyse. À l'aide d'un séparateur «Folsom Splitter », les échantillons ont été divisés en trois parties dont une partie $(100 \mathrm{ml})$ a servi à l'analyse de la biomasse du zooplancton. La biomasse du zooplancton a été estimée par le poids organique des particules retenues par filtration séquentielle sur une série de tamis en filet NITEX ${ }^{\mathrm{TM}}$ de maille décroissante $(500,200,100$ et 
$53 \mu \mathrm{m})$. Les particules récoltées sur chacun des tamis ont été recueillies sur un filtre Whatmann GF/C (27 mm diamètre et 1,2 $\mu \mathrm{m}$ de porosité moyenne) dans des colonnes à filtration Millipore. Les filtres et leurs résidus ont été séchés à $60^{\circ} \mathrm{C}$ pendant 18 heures et pesés sur une microbalance Cahn $( \pm 0,1 \mu \mathrm{g})$ afin d'obtenir la masse sèche de matière. Ces mêmes filtres ont ensuite été brûlés à $500^{\circ} \mathrm{C}$ durant 18 heures et les filtres avec les résidus cendrés ont été pesés pour estimer la masse des résidus minéraux. Finalement, le poids des cendres (résidus minéraux) a été soustrait du poids sec (masse sèche de matière) afin d'obtenir la masse de matière organique, soit le poids en masse sèche sans cendres (Ash-free dry weight, AFDW) par volume d'eau filtré $\left(\mathrm{mg} \cdot \mathrm{m}^{-3}\right.$ ) pour chacune des classes de taille $(53-100,100-200,200-500$ et $>500 \mu \mathrm{m})$. Les classes de taille de zooplancton correspondent à des particules comprenant des détritus et des algues en plus du zooplancton, en particulier dans les deux dernières classes de taille $<200 \mu \mathrm{m}$. La biomasse du zooplancton total correspond à la somme des masses de matière organique dans chaque classe de taille.

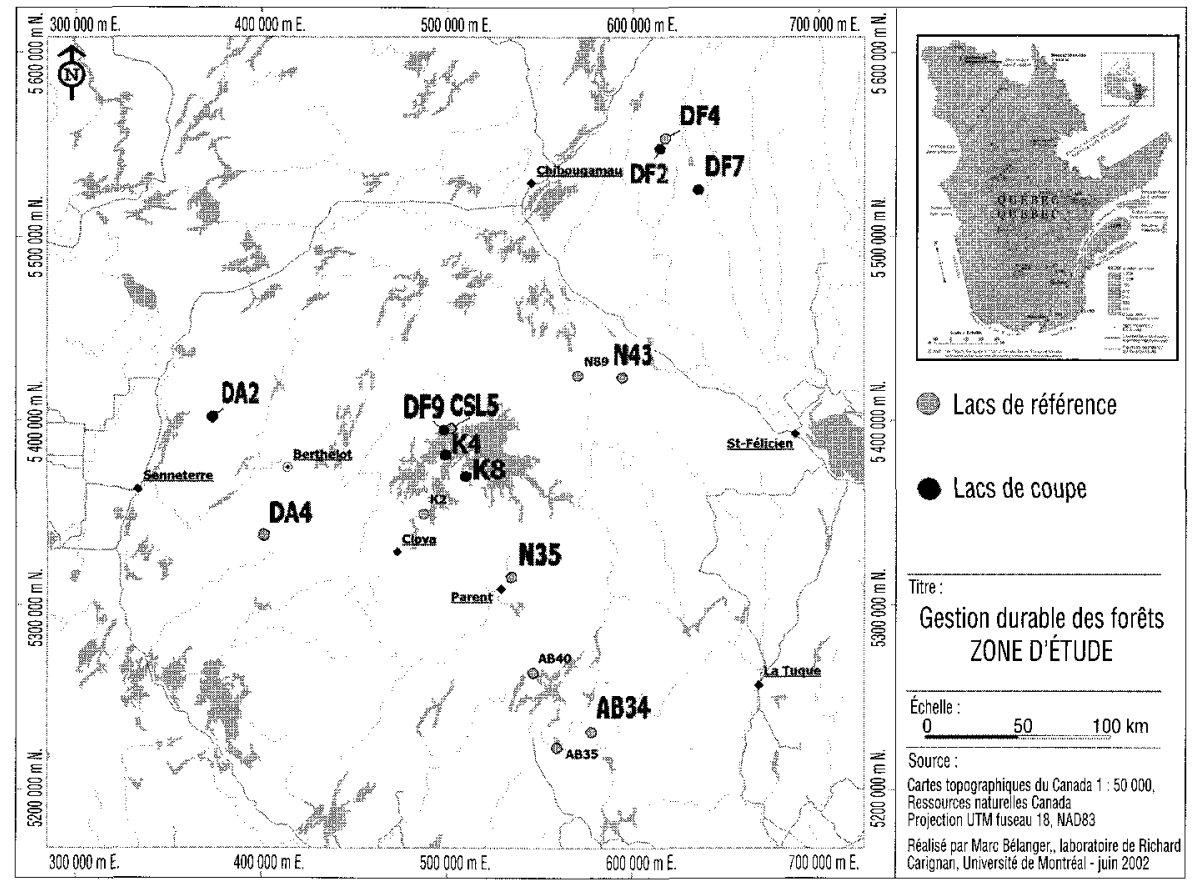

Figure 2 Positions géographiques des dix lacs de référence (gris: $A B 34$, AB35, AB40, CSL5, DA4, DF4, N35, N43, N89, K2) et des six lacs de coupe (noir: DA2, DF2, DF7, DF9, K4, K8) dans la région d'étude. Les six lacs de référence sélectionnés (AB34, CSL5, DA4, DF4, N35, N43) sont en caractères gras. Les quatre lacs exclus par la méthode de sélection (AB35, AB40, N89, K2) sont en petits caractères.

Geographical locations of the ten reference lakes (grey: $A B 34, A B 35$, $A B 40, C S L 5, D A 4, D F 4, N 35, N 43, N 89, K 2)$ and the six cut lakes with important harvesting (black: DA2, DF2, DF7, DF9, K4, K8) in the study area. The six reference lakes selected in the study (AB34, CSL5, DA4, $D F 4, N 35, N 43)$ are in bold letters. The four lakes excluded by the selection (AB35, AB40, N89, K2) are in small letters. 


\subsection{Analyses statistiques}

Trois analyses en composantes principales (ACP) ont permis de décrire la variance des 16 lacs à l'étude selon leur morphométrie, la qualité de l'eau, et la biomasse des quatre classes de taille du zooplancton dans la colonne d'eau avant la coupe (juin 2000 ; voir figures $3 a$, $4 a$ et $5 a$ ), d'identifier quelles variables (descripteurs) expliquaient la plus grande partie de cette variance et de visualiser quels descripteurs étaient corrélés ensemble (voir figures 3b, 4b et $5 \mathrm{~b}$ ). Les ordinations ont aussi permis de visualiser la distribution des lacs selon les vecteurs des descripteurs et de sélectionner les six lacs de référence les plus semblables aux six lacs de coupe (ayant des positions rapprochées dans le plan d'ordination).

Pour ces trois ACPs, les vecteurs propres ont été normés à 1 afin de préserver la distance euclidienne entre les objets et de déterminer le cercle de contribution équilibrée. Les descripteurs sortant du cercle de contribution équilibrée étaient ceux ayant une grande contribution à la formation des deux premiers axes de l'ordination et à la distribution des lacs dans cet espace (LEGENDRE et LEGENDRE, 1998) (figures 3b, 4b, 5b). Les trois ACPs ont été calculées avec le Progiciel R selon LEGENDRE et LEGENDRE (1998). Les coordonnées des objets et des vecteurs propres ont été exportées vers SigmaPlot 2000 afin de tracer les graphiques d'ordination.

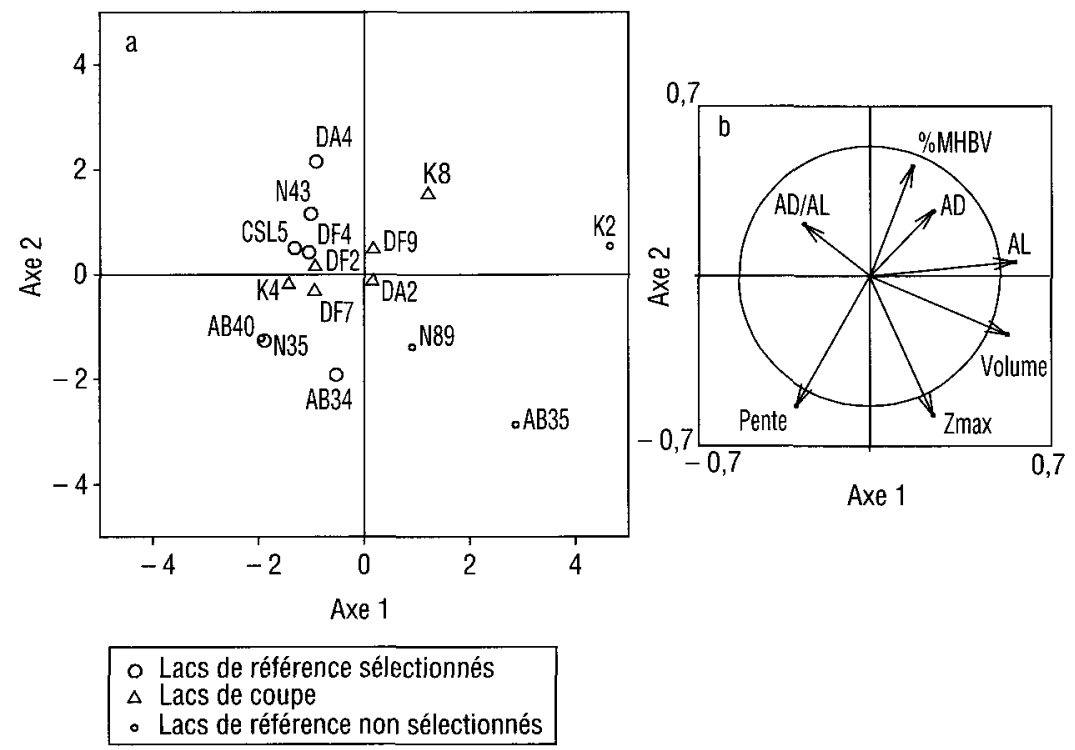

Figure 3 a : Diagramme d'ordination des dix lacs de référence et des six lacs de coupe d'après une analyse en composantes principales basée sur les descripteurs de la morphométrie; $b$ : projection des vecteurs propres des descripteurs et du cercle de contribution équilibrée (rayon $=0,5345$ ).

a: Ordination diagram of the ten reference lakes and the six cut lakes according to a principal component analysis based on the morphometric descriptors; $b$ : projection of eigenvectors of descriptors and the equilibrium circle (radius $=0.5345$ ). 


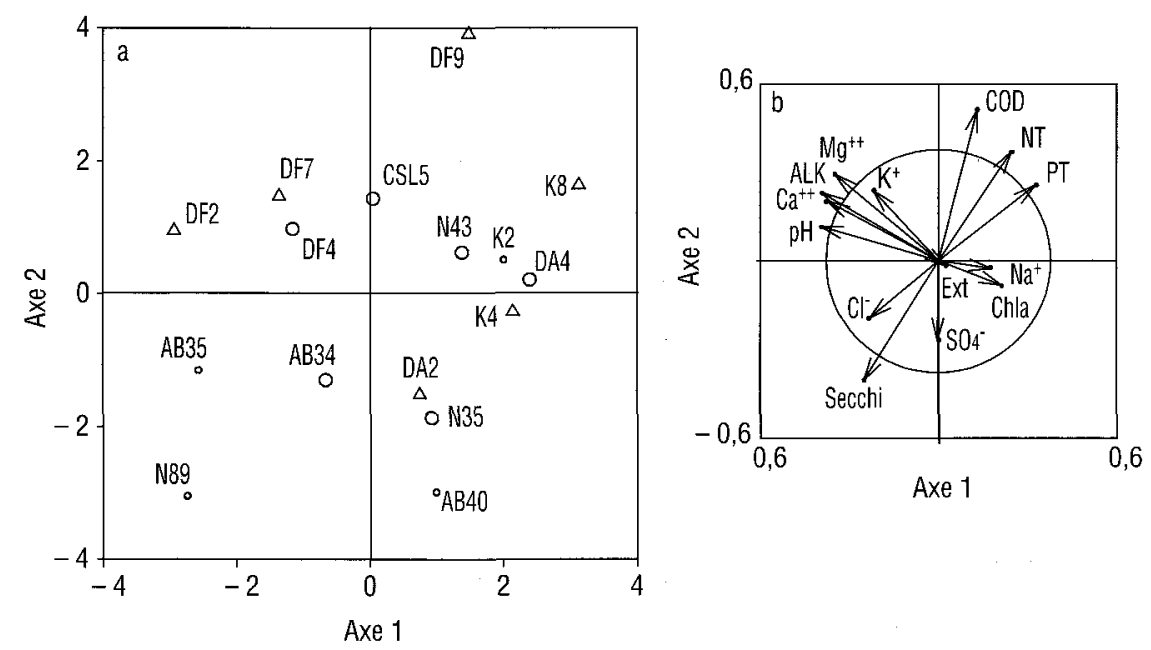

- Lacs de référence sélectionnés

$\triangle$ Lacs de coupes

- Lacs de référence non sélectionnés

Figure 4 a : Diagramme d'ordination des dix lacs de référence et des six lacs de coupe d'après une analyse en composantes principales basée sur les descripteurs de la qualité des eaux; $b$ : projection des vecteurs propres des descripteurs et du cercle de contribution équilibrée (rayon $=0,3780$ ).

a: Ordination diagram of the ten reference lakes and the six cut lakes according to a principal component analysis based on the water quality descriptors; $b$ : projection of eigenvectors of the descriptors and the equilibrium circle (radius $=0.3780$ ).

Les analyses de redondance (RDA) ont servi à tester si les deux groupes de lacs (lacs de coupe et lacs de référence sélectionnés) étaient semblables quant aux variables de la morphométrie, de la qualité de l'eau et du zooplancton. Pour chacune des analyses de redondance, la matrice $X$ (perturbation) était composée d'une variable binaire permettant de différencier les lacs de coupe des lacs de référence. La matrice $Y$ (descripteurs) comprenait un des groupes de variables quantitatives : morphométrie, qualité de l'eau ou classes de taille du zooplancton (tableau 1). 


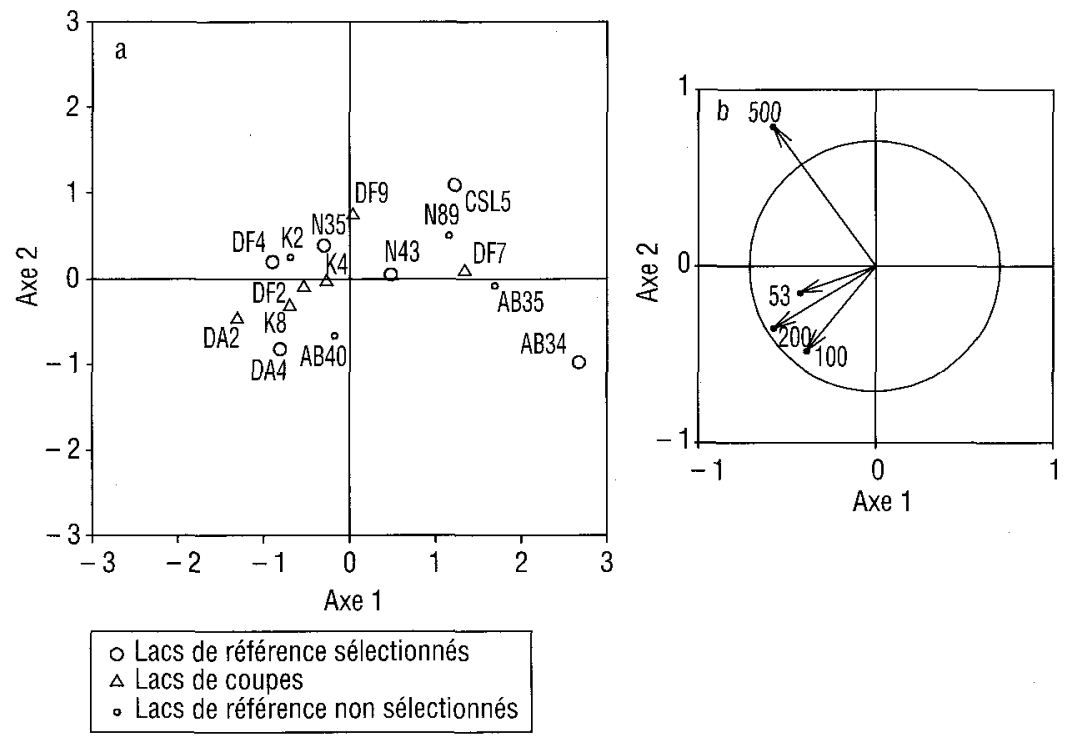

Figure 5 a : Diagramme d'ordination des dix lacs de référence et des six lacs de coupe d'après une analyse en composantes principales basée sur les descripteurs du zooplancton ; $b$ : projection des vecteurs propres des descripteurs et du cercle de contribution équilibrée (rayon = 0,7071 ).

a: Ordination diagram of the ten reference lakes and the six cut lakes according to a principal component analysis based on the zooplankton descriptors; $b$ : projection of eigenvectors of the descriptors and the equilibrium circle (radius $=0.7071$ ).

Les valeurs des trois groupes de variables ont été transformées en leur logarithme à base dix pour avoir des distributions symétriques et des relations linéaires entre les variables (LEGENDRE et LEGENDRE, 1998). Les tests statistiques ont été faits par 9999 permutations sans restriction sur la permutation des lignes. Les analyses ont été faites sur la matrice de covariance avec les vecteurs propres normalisés à 1 comme suggéré par LEGENDRE et LEGENDRE (1998). Toutes les RDAs ont été faites avec CANOCO 4.0. (TER BRAAK et SMILAUER, 1998). Pour ces analyses, l'hypothèse nulle prévoyait l'indépendance des matrices $Y$ et $X$. Lorsque le test par permutation se révélait significatif pour l'ensemble des axes canoniques $(P<0,05)$, la matrice $X$ expliquait la matrice $Y$. Dans un tel cas, les variables de la matrice $Y$ étaient séparées en deux groupes distincts comme dans la matrice des variables explicatives binaires (X). Ceci signifiait que les descripteurs (morphométrie, qualité de l'eau, zooplancton) étaient significativement différents entre les lacs de coupe et les lacs de référence sélectionnés. Si le test par permutation n'était pas significatif $(P>0,05)$, ceci signifiait que les lacs de coupe et les lacs de référence étaient similaires au niveau de leurs descripteurs et qu'ils pouvaient être utilisés dans le cadre d'une étude d'impact basée sur l'approche BACI. 
Tableau 1 Analyses de redondance (RDA) avec la matrice $X$ (perturbation: variable binaire) et les matrices $Y$ (descripteurs : morphométrie, qualité de l'eau, zooplancton: variables quantitatives) pour tester si les caractéristiques des lacs de référence sélectionnés sont similaires à celles des lacs de coupe.

Table 1 Redundancy analysis (RDA) with matrix $X$ (perturbation: binary variable) and matrices $Y$ (descriptors: morphometry, water quality, zooplankton: quantitative variables) to test if limnological characteristics of the selected reference lakes are similar to those of the cut lakes.

\begin{tabular}{|c|c|c|c|c|c|c|c|c|c|c|}
\hline \multirow{2}{*}{\multicolumn{3}{|c|}{$\begin{array}{c}\begin{array}{c}\text { Matrice } \\
\text { de perturbation }\end{array} \\
\begin{array}{c}\text { Variable } \\
\text { binaire }\end{array} \\
\end{array}$}} & \multicolumn{8}{|c|}{ Matrices des descripteurs } \\
\hline & & & \multicolumn{3}{|c|}{$\begin{array}{c}\text { Variables } \\
\text { morphométrie }\end{array}$} & \multicolumn{3}{|c|}{$\begin{array}{c}\text { Variables } \\
\text { qualité de l'eau }\end{array}$} & \multicolumn{2}{|c|}{$\begin{array}{l}\text { Variables } \\
\text { zooplancton }\end{array}$} \\
\hline & Lacs & Groupe & Lacs & Pente & AL... & Lacs & PT & $\operatorname{COD} \ldots$ & Lacs & $500 \mu \mathrm{m} 200 \mu \mathrm{m} .$. \\
\hline \multirow{6}{*}{ 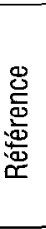 } & AB34 & 1 & $\mathrm{AB} 34$ & & & AB34 & & & $\overline{A B 34}$ & \\
\hline & CSL5 & 1 & CSL5 & & & CSL5 & & & CSL5 & \\
\hline & DA4 & 1 & DA4 & & & DA4 & & & DA4 & \\
\hline & DF4 & 1 & DF4 & & & DF4 & & & DF4 & \\
\hline & N35 & 1 & N35 & & & N35 & & & N35 & \\
\hline & N43 & 1 & $\mathrm{~N} 43$ & & & N43 & & & $\mathrm{N} 43$ & \\
\hline \multirow{6}{*}{$\begin{array}{l}\text { 응 } \\
\text { 잉 }\end{array}$} & $\mathrm{DA} 2$ & 0 & $\mathrm{DA} 2$ & & & $\mathrm{DA} 2$ & & & DA2 & \\
\hline & DF2 & 0 & DF2 & & & DF2 & & & DF2 & \\
\hline & DF7 & 0 & DF7 & & & DF7 & & & DF7 & \\
\hline & DF9 & 0 & DF9 & & & DF9 & & & DF9 & \\
\hline & K4 & 0 & K4 & & & K4 & & & K4 & \\
\hline & K8 & 0 & K8 & & & K8 & & & K8 & \\
\hline
\end{tabular}

\section{3 - RÉSULTATS ET DISCUSSION}

\subsection{Comparaison et sélection des lacs selon leur morphométrie}

Les 16 lacs à l'étude présentent une variabilité naturelle entre les groupes de lacs au niveau de leur morphométrie (tableau 2). En moyenne, les lacs de référence ont une surface de $0,46 \mathrm{~km}^{2}$ pour une aire de drainage de $2,94 \mathrm{~km}^{2}$, ce qui correspond à un ratio de drainage de 7,92 . Leur volume est de $2 \cdot 10^{6} \mathrm{~m}^{3}$ et leur profondeur maximale de $11,57 \mathrm{~m}$. La pente moyenne du bassin versant des lacs de référence est de $10 \%$ et le pourcentage de milieux humides est faible $(0,03 \%)$. En moyenne, la surface des lacs de coupe $\left(0,41 \mathrm{~km}^{2}\right)$ et de leur bassin versant $\left(2,23 \mathrm{~km}^{2}\right)$ est similaire à celles des lacs de référence mais le ratio de drainage est plus faible $(5,18)$. La pente du bassin versant $(6,93 \%)$, le volume $\left(1 \cdot 10^{6} \mathrm{~m}^{3}\right)$ et la profondeur maximale $(9,22 \mathrm{~m})$ des lacs de coupe sont aussi en moyenne plus faibles que pour les lacs de référence pour lesquels les maximums sont plus élevés.

Les variables ayant une forte contribution à la variance totale dans la morphométrie des lacs et de leurs bassins versants (celles sortant du cercle de contribution équilibrée de rayon $=0,5345$ ) sont le volume, la profondeur maximale et l'aire du lac, ainsi que la pente du bassin versant (figure $3 b$ ). Le premier 
axe de l'ordination explique $38,4 \%$ de la variance totale alors que le deuxième axe en explique $25,6 \%$ et les deux axes sont significatifs et peuvent être interprétés (méthode du bâton brisé : $1^{\text {er }}$ axe $=37,0 \% ; 2^{\mathrm{e}}$ axe $=22,8 \%$ ). Le premier axe semble expliqué davantage par l'aire et le volume du lac alors que le deuxième axe l'est plutôt par la pente du bassin versant et la profondeur maximale du lac (figure $3 b$ ). Le diagramme des vecteurs propres (descripteurs) montre qu'il y a une corrélation positive (angle faible entre les descripteurs) entre la pente du bassin versant et la profondeur maximale du lac d'une part, et entre l'aire et le volume du lac, d'autre part.

Tableau 2 Valeurs des variables morphométriques dans chacun des lacs et moyenne, écart-type, minimum et maximum pour les dix lacs de référence et les six lacs de coupe. Pente : pente du bassin versant, $A L$ : aire du lac, $A D$ : aire de drainage, $A D / A L$ : ratio de drainage, Volume : volume du lac, Zmax : profondeur maximale, \%MHBV : \% de milieux humides sur le bassin versant. Les données de morphométrie proviennent du laboratoire de M. Carignan de l'Université de Montréal.

Tableau 2 Values of morphometric variables in each lake and mean, standard deviation, minimum and maximum for the ten reference lakes and the six cut lakes. Slope: slope of the watershed, AL: lake area, AD: drainage area, $A D / A L$ : drainage ratio, Volume: lake volume, Zmax: maximal depth, \%MHBV: \% of wetlands in the watershed. Morphometric data were obtained from the laboratory of Dr. Carignan at the Université de Montréal.

\begin{tabular}{|c|c|c|c|c|c|c|c|}
\hline $\begin{array}{c}\text { Lacs } \\
\text { de référence } \\
\end{array}$ & $\begin{array}{c}\text { Pente } \\
(\%)\end{array}$ & $\begin{array}{c}\mathrm{AL} \\
\left(\mathrm{km}^{2}\right)\end{array}$ & $\begin{array}{c}\mathrm{AD} \\
\left(\mathrm{km}^{2}\right)\end{array}$ & $\mathrm{AD} / \mathrm{AL}$ & $\begin{array}{c}\text { Volume } \\
\left(\mathrm{m}^{3}\right)\end{array}$ & $\underset{(m)}{Z \max }$ & $\begin{array}{c}\% \text { MHBV } \\
(\%)\end{array}$ \\
\hline AB34 & 14,5 & 0,27 & 3,2 & 11,0 & 2178386 & 17,8 & 0,004 \\
\hline AB35 & 9,5 & 0,87 & 1,8 & 2,0 & 5585714 & 21,7 & 0,007 \\
\hline AB40 & 15,5 & 0,18 & 1,0 & 5,6 & 566382 & 9,1 & 0,000 \\
\hline CSL5 & 8,7 & 0,15 & 2,2 & 10,4 & 813748 & 9,2 & 0,055 \\
\hline DA4 & 6,3 & 0,26 & 3,6 & 13,8 & 773820 & 6,7 & 0,129 \\
\hline DF4 & 7,8 & 0,30 & 1,7 & 5,5 & 778091 & 6.4 & 0,013 \\
\hline K2 & 4,6 & 1,42 & 7,7 & 5,3 & 6144649 & 12,2 & 0,044 \\
\hline N35 & 18,1 & 0,21 & 1,1 & 5,4 & 648179 & 9,1 & 0,043 \\
\hline N43 & 7,6 & 0,30 & 5,1 & 17,2 & 921314 & 9,5 & 0,019 \\
\hline N89 & 10,7 & 0,67 & 2,0 & 3,0 & 2910787 & 14,0 & 0,017 \\
\hline Moyenne & 10,3 & 0,46 & 2,9 & 7,9 & 2132107 & 11,6 & 0,033 \\
\hline Écart-type & 4,4 & 0,41 & 2,1 & 4,9 & 2112721 & 5,0 & 0,039 \\
\hline Minimum & 4,6 & 0,15 & 1,0 & 2,0 & 566382 & 6,4 & 0,000 \\
\hline Maximum & 18,1 & 1,42 & 7,7 & 17,2 & 6144649 & 21,7 & 0,129 \\
\hline $\begin{array}{c}\text { Lacs } \\
\text { de coupe }\end{array}$ & $\begin{array}{c}\text { Pente } \\
(\%)\end{array}$ & $\underset{\left(\mathrm{km}^{2}\right)}{\mathrm{AL}}$ & $\underset{\left(\mathrm{km}^{2}\right)}{A D}$ & $\mathrm{AD} / \mathrm{AL}$ & $\begin{array}{c}\text { Volume } \\
\left(\mathrm{m}^{3}\right)\end{array}$ & $\underset{(\mathrm{m})}{\mathrm{Zmax}}$ & $\begin{array}{c}\% \text { MHBV } \\
(\%)\end{array}$ \\
\hline DA2 & 8,4 & 0,51 & 2,2 & 4,4 & 1754202 & 10,7 & 0,045 \\
\hline DF2 & 6,8 & 0,29 & 0,9 & 2,9 & 844038 & 6,7 & 0,000 \\
\hline DF7 & 10,5 & 0,32 & 2,7 & 8,6 & 966594 & 10,8 & 0,008 \\
\hline DF9 & 3,3 & 0,42 & 1,8 & 4,3 & 1398745 & 10,5 & 0,032 \\
\hline K4 & 9,6 & 0,19 & 0,9 & 4,5 & 558582 & 8,0 & 0,017 \\
\hline K8 & 3,0 & 0,76 & 4,9 & 6,4 & 1749715 & 8,6 & 0,052 \\
\hline Moyenne & 6,9 & 0,41 & 2,2 & 5,2 & 1211979 & 9,2 & 0,026 \\
\hline Écart-type & 3,2 & 0,12 & 1,5 & 2,0 & 498110 & 1,7 & 0,021 \\
\hline Minimum & 3,0 & 0,19 & 0,9 & 2,9 & 558582 & 6,7 & 0,000 \\
\hline Maximum & 10,5 & 0,76 & 4,9 & 8,6 & 1754202 & 10,8 & 0,052 \\
\hline
\end{tabular}


Les six lacs de coupe (DA2, DF2, DF7, DF9, K4, K8) se trouvent au centre de la distribution des lacs dans le graphique d'ordination. Seul le lac de coupe K8 est un peu excentrique avec une pente du bassin versant plus faible et une aire et un volume plus grands comparés aux autres lacs de coupe (figure 3a). Parmi les lacs de référence, ceux qui diffèrent le plus des lacs de coupe au niveau de leur morphométrie sont les lacs $K 2$ et $A B 35$. Le lac K2 est extrême avec des valeurs très élevées quant à l'aire et au volume du lac alors que le lac AB35 a un volume et une profondeur maximale plus élevés comparativement aux lacs de coupe et aux autres lacs de référence (AB34, AB40, CSL5, DA4, DF4, N35, N89 et N43). II serait alors préférable de ne pas les sélectionner comme lacs de référence. Les autres lacs de référence semblent distribués de façon similaire sur le premier axe. Pour le deuxième axe, certains lacs de référence semblent avoir des valeurs un peu plus élevées pour la pente du bassin versant (AB34, AB40, N35) ou la profondeur maximale du lac (N89) comparativement aux lacs de coupe. Si la distribution de ces lacs est également différente de celle des lacs de coupe pour les autres descripteurs (qualité de l'eau et zooplancton), il serait justifié de ne pas les sélectionner comme lacs de référence.

Il est crucial de tenir compte des variations entre les lacs dans les variables morphométriques car des différences importantes dans la morphométrie des lacs de coupe et des lacs de référence pourraient confondre les résultats de l'analyse BACl. En effet, l'effet des coupes forestières pourrait être amplifié ou amoindri selon le type de morphométrie des lacs et des bassins versants. Ainsi, les lacs de la forêt boréale ayant un grand ratio de drainage (AD/AL ou $A D / \mathrm{Nol}$, où $A D=$ aire de drainage, $A L=$ aire du lac, et Vol = volume du lac), c'est-à-dire un grand bassin de drainage relativement à la surface ou au volume du lac, ont un taux d'exportation en nutriments et en carbone organique plus élevé (LAMONTAGNE et al., 2000) et présentent des concentrations plus fortes de PT et de COD (variables susceptibles de modifier la communauté zooplanctonique) après des coupes à blanc sur leur bassin versant (GARCIA et CARIGNAN, 1999 ; CARIGNAN et al., 2000) que ceux ayant un faible ratio de drainage $(<4)$. Dans les lacs de la plaine boréale, ceux ayant un grand ratio de drainage (AD/Vol) présentent aussi les plus fortes concentrations de PT après des coupes forestières (PREPAs et al., 2001). De plus, D'ARCY et CARIGNAN (1997) ont montré que même dans les lacs naturels du bouclier canadien, la pente du bassin versant et la morphométrie du lac expliquent de 50 à $70 \%$ de la variabilité en $\mathrm{PT}, \mathrm{COD}$ et $\mathrm{Chl}$ a, variables qui ont toutes une relation négative avec la pente. En fait, la pente, la superficie drainée et le pourcentage de milieu humide dans le bassin versant prédisent aussi bien la Chl a que le PT. De plus, la pente et le pourcentage de milieux humides peuvent avoir des effets sur les apports en COD et en PT. En fait, les concentrations de COD et de PT diminuent dans les lacs dont le bassin versant a une forte pente car la vitesse de percolation de l'eau est plus rapide, ce qui diminue le contact de l'eau avec l'horizon superficiel de matière organique et permet la rétention des nutriments dans la solution du sol dans les horizons minéraux en profondeur. Dans ces conditions, l'eau concentre moins de matière organique (et en perd dans le sol minéral), ce qui diminue la quantité de COD dans les eaux de surface des lacs (HOULE et al., 1995). À l'opposé, des bassins versants de faible topographie et riches en milieux humides favorisent un écoulement des eaux en surface et le transport direct du COD et du PT dans les eaux des lacs. 


\subsection{Comparaison et sélection des lacs selon la qualité de l'eau}

Le tableau 3 présente les valeurs des variables de la qualité de l'eau dans chaque lac et en moyenne pour les lacs de coupe et les lacs de référence. Dans l'ensemble, les lacs à l'étude sont des lacs mésotrophes avec des concentrations respectives en phosphore total et en chlorophylle a (Chl a) d'environ 10 et $2 \mu \mathrm{g} \cdot \mathrm{L}^{-1}$. Les eaux sont légèrement acides avec un $\mathrm{pH}$ moyen de 6,4 et assez colorées avec une transparence au Secchi de 2 à $3 \mathrm{~m}$ et des concentrations de carbone organique dissous (COD) de 6 à $9 \mathrm{mg} \cdot \mathrm{L}^{-1}$. II existe une assez forte variation de la qualité de l'eau entre les lacs, avec un écart entre les valeurs minimum et maximum d'un facteur 2 à 10.

Les variables ayant une forte contribution à la variance totale dans la qualité de l'eau sont celles qui sortent du cercle de contribution équilibrée (rayon = 0,3780 ), soit le phosphore total (PT), l'azote total (NT), le carbone organique dissous (COD), le Secchi, $\mathrm{Ca}^{2+}, \mathrm{Mg}^{2+}$, l'alcalinité (ALK) et le $\mathrm{pH}$ (figure 4b). Le premier axe, associé à $\mathrm{ALK}, \mathrm{Ca}^{++}, \mathrm{Mg}^{++}$et au $\mathrm{pH}$, explique $36,4 \%$ de la variance totale et le deuxième axe, associé au COD, PT et à NT en explique $22,4 \%$. Ces deux axes sont significatifs et interprétables (méthode du bâton brisé : $23,2 \%$ et $16,1 \%$ ) (figure $4 b$ ).

Dans le diagramme des vecteurs propres des descripteurs de la qualité de l'eau (figure 4b), on remarque que le COD est positivement corrélé avec NT et PT, tel que démontré dans plusieurs autres études (BAYLEY et al., 1992 ; SCHINDLER et al., 1992). Tel qu'attendu, la transparence au Secchi est négativement corrélée au COD (JONES, 1992) et aux nutriments (PT, NT ; angle près de $180^{\circ}$ ), qui diminuent tous les deux la pénétration de la lumière. Finalement, la Chl a ne semble pas avoir de corrélation avec les nutriments (PT, NT et COD) dans les deux premiers axes de l'ordination, bien que ces variables soient reconnues comme étant corrélées dans de nombreuses autres études (SAKAMOTO, 1966 ; DILLON et RIGLER, 1974 ; SCHINDLER, 1978 ; PACE, 1984 ; MCQUEEN et al., 1986 ; CURRIE et al., 1999 ; DEL GIORGIO et al., 1994 ; 1997). Les projections des troisième et quatrième axes avec les deux premiers ne démontrent pas davantage de corrélation entre ces variables (Chl a vs PT, NT et COD).

Parmi les lacs de coupe, les lacs DF2 et DF7 sont caractérisés par des valeurs de $\mathrm{pH}, \mathrm{ALK}, \mathrm{Ca}^{++}$et $\mathrm{Mg}^{++}$plus élevées comparées à celles des autres lacs (figure 4a) alors que les lacs DA2 et K4 ont des valeurs plus faibles pour ces quatre variables. Deux autres lacs de coupe, les lacs K8 et DF9, semblent également en marge de la distribution avec de grandes concentrations en COD, PT et NT et une faible transparence au Secchi. Les lacs de référence qui se distinguent le plus des lacs de coupe et des autres lacs de référence au niveau de la qualité des eaux sont les lacs AB35, N89 et AB40 (figure 4a). Les lacs de référence $A B 35$ et N89 ont une position contraire aux lacs de coupe K8 et DF9, avec une grande transparence au Secchi et de faibles concentrations de COD, PT et NT. Le lac de référence AB40 diffère des lacs de coupe par des valeurs plus faibles de $\mathrm{pH}$, d'ALK, de $\mathrm{Ca}++$ et de $\mathrm{Mg}++$. En définitive, il ressort du diagramme d'ordination que les lacs AB35, N89 et AB40 ne devraient pas être sélectionnés comme lacs de référence car trop différents des lacs de coupe au niveau de la qualité des eaux. Parmi ces trois lacs, le lac AB35 avait déjà été éliminé sur la base des différences dans la morphométrie. 
Tableau 3 Valeurs des variables de la qualité de l'eau dans chacun des lacs de coupe et moyenne, écart-type, minimum et maximum pour les dix lacs de référence et les six lacs de coupe : Secchi : transparence de l'eau, Ext : coefficient d'extinction de la lumière, $\mathrm{COD}$ : carbone organique dissous, PT : phosphore total, NT : azote total, $\mathrm{pH}$ : $\mathrm{pH}$, ALK : alcalinité, $\mathrm{SO}^{-}$: sulfates, $\mathrm{Cl}^{-}$: chlorures, $\mathrm{Na}^{+}$: sodium, $\mathrm{Mg}^{++}$: magnésium, $\mathrm{Ca}^{++}$: calcium, $\mathrm{K}^{+}$: potassium, $\mathrm{Chl}-\mathrm{a}$ : chlorophylle-a. Les données de qualité de l'eau proviennent du laboratoire de $\mathrm{M}$. Carignan de l'Université de Montréal.

Table 3 Values of water quality variables in each cut lake and mean, standard deviation, minimum and maximum for the ten reference lakes and the six cut lakes. Secchi: water transparency, Ext: light extinction coefficient, COD: dissolved organic carbon, PT: total phosphorus, NT: total nitrogen, $\mathrm{pH}: \mathrm{pH}, \mathrm{ALK}$ : alkalinity, SO4: sulfates, $\mathrm{Cl}^{-}$: chlorides, $\mathrm{Na}^{+}$: sodium, $\mathrm{Mg}^{++}$: magnesium, $\mathrm{Ca}^{++}$: calcium, $\mathrm{K}^{+}$: potassium, $\mathrm{Chl}$-a: chlorophyll-a. Physico-chemical data were obtained from the laboratory of Dr. Carignan at the Université de Montréal.

\begin{tabular}{|c|c|c|c|c|c|c|c|c|c|c|c|c|c|c|}
\hline $\begin{array}{l}\text { Lacs de } \\
\text { référence }\end{array}$ & $\begin{array}{l}\text { Secchi } \\
\text { (m) }\end{array}$ & $\begin{array}{c}\text { Ext } \\
\left(m^{-1}\right)\end{array}$ & $\begin{array}{c}\text { COD } \\
(\mathrm{mg} / \mathrm{L})\end{array}$ & $\begin{array}{c}\text { PT } \\
(\mu \mathrm{g} / \mathrm{L})\end{array}$ & $\begin{array}{c}\text { NT } \\
(\mu \mathrm{g} / \mathrm{L})\end{array}$ & $\mathrm{pH}$ & $\begin{array}{c}\text { ALK } \\
(\mu \mathrm{eq} / \mathrm{L})\end{array}$ & $\begin{array}{c}\mathrm{SO4}^{-} \\
(\mathrm{mg} / \mathrm{h})\end{array}$ & $\begin{array}{c}\mathrm{Cl}^{-} \\
(\mathrm{mg} / \mathrm{L})\end{array}$ & $\begin{array}{c}\mathrm{Na}^{+} \\
(\mathrm{mg} / \mathrm{L})\end{array}$ & $\begin{array}{c}\mathrm{Mg}^{2+} \\
(\mathrm{mg} / \mathrm{L})\end{array}$ & $\begin{array}{c}\mathrm{Ca}^{2+} \\
(\mathrm{mg} / \mathrm{L})\end{array}$ & $\begin{array}{c}\mathbf{K}^{+} \\
(\mathrm{mg} / \mathrm{L})\end{array}$ & $\begin{array}{r}\text { Chl-a } \\
(\mu \mathrm{g} / \mathrm{L})\end{array}$ \\
\hline AB34 & 3,20 & 1,08 & 5,31 & 8,17 & 262 & 6,49 & 83 & 1,57 & 0,18 & 0,64 & 0,57 & 2,96 & 0,34 & 3,73 \\
\hline AB35 & 4,80 & 0,88 & 5,39 & 7,85 & 245 & 6,55 & 86 & 1,30 & 0,20 & 0,46 & 0,56 & 2,73 & 0,50 & 1 \\
\hline$A B 40$ & 4,00 & 0,75 & 3,09 & 9,40 & 206 & 6,15 & 29 & 0,94 & 0,14 & 0,46 & 0,30 & 1,22 & 0,11 & 2,67 \\
\hline CSL5 & 1,95 & 2,01 & 9,71 & 9,40 & 292 & 6,63 & 93 & 0,85 & 0,14 & 0,79 & 0,63 & 2,36 & 0,40 & 2,37 \\
\hline DA4 & 1,00 & 2,75 & 8,86 & 11,23 & 256 & 5,81 & 15 & 0,86 & 0,12 & 0,54 & 0,36 & 1,16 & 0,22 & 1,59 \\
\hline DF4 & 2,20 & 1,57 & 9,26 & 9,40 & 250 & 6,74 & 93 & 0,47 & 0,18 & 0,36 & 0,65 & 2,06 & 0,30 & 1,76 \\
\hline K2 & 1,80 & 2,05 & 8,65 & 11,10 & 332 & 5,98 & 17 & 0,77 & 0,14 & 0,49 & 0,42 & 1,29 & 0,22 & 1,70 \\
\hline N35 & 3,90 & 0,92 & 4,29 & 10,80 & 219 & 6,27 & 41 & 0,73 & 0,12 & & 0,41 & 1,16 & 0,11 & 1,78 \\
\hline N43 & 2,00 & 1,88 & 8,02 & 10,55 & 301 & 6,43 & 54 & 0,5 & 0 & & 0,46 & 1,38 & 0,21 & 1,59 \\
\hline N89 & 4,70 & 0,79 & & 5,10 & 155 & 6,91 & 87 & 0, & 0 , & & 0,47 & 1, & 0,23 & 1,49 \\
\hline Moye & 2,96 & 1,47 & & 9,30 & 252 & 6,39 & 6 & & & & 48 & & 0,26 & 2,01 \\
\hline t-type & 1,34 & 0,69 & 2.5 & 1,88 & 51 & 0,34 & 3 & 0,3 & 0,0 & 0 & 0,12 & 0.68 & 0.12 & 0,72 \\
\hline Vinimum & 1,00 & 0,75 & 3,09 & 5,10 & 155 & 5,81 & 1 & 0,4 & 0,1 & 0,3 & 0,30 & 1,16 & 0,11 & 1,46 \\
\hline Maximum & 4,80 & 2,75 & 9,71 & 11,23 & 332 & 6,91 & 93 & 1,57 & 0,27 & 0,79 & 0,65 & 2,96 & 0,50 & 3,73 \\
\hline $\begin{array}{l}\text { Lacs de } \\
\text { rélérence }\end{array}$ & $\begin{array}{l}\text { Secchi } \\
\text { (m) }\end{array}$ & $\begin{array}{c}\text { Ext } \\
\left(m^{-1}\right)\end{array}$ & $\begin{array}{c}\mathrm{COD} \\
(\mathrm{mg} / \mathrm{L})\end{array}$ & $\begin{array}{c}P T \\
(\mu g / L)\end{array}$ & $\begin{array}{c}\text { NT } \\
(\mu \mathrm{g} / \mathrm{L})\end{array}$ & $\mathrm{pH}$ & $\begin{array}{c}\text { ALK } \\
(\mu \mathrm{eq} / \mathrm{L})\end{array}$ & $(\mathrm{mg} / \mathrm{L})$ & $\begin{array}{c}\mathrm{Cl}^{-} \\
(\mathrm{mg} / \mathrm{L})\end{array}$ & $\begin{array}{c}\mathrm{Na}^{+} \\
(\mathrm{mg} / \mathrm{L})\end{array}$ & $\begin{array}{c}\mathrm{Mg}^{2+} \\
(\mathrm{mg} / \mathrm{L})\end{array}$ & $\begin{array}{c}\mathrm{Ca}^{2+} \\
(\mathrm{mg} / \mathrm{L})\end{array}$ & $\begin{array}{c}\mathbf{K}^{+} \\
(\mathbf{m g} / \mathrm{L})\end{array}$ & $\begin{array}{l}\text { Chl-a } \\
(\mu \mathrm{g} / \mathrm{L})\end{array}$ \\
\hline DA2 & 2.90 & 1,20 & 6.56 & 7.33 & 234 & 6,38 & 18 & 0.96 & 0.12 & 0,52 & 0,30 & 1,22 & 0,14 & 1,39 \\
\hline DF2 & 2.90 & 1,25 & 6.76 & 6.80 & 224 & 7.00 & 171 & 0.4 & 0.1 & 0.3 & 0.39 & 2.66 & 0.29 & 1.92 \\
\hline DF7 & 2,30 & 1,72 & 9,95 & 8,10 & 221 & 6,77 & 128 & 0,6 & 0,1 & 0,49 & 0,96 & 2,40 & 0,28 & 1,89 \\
\hline DF9 & 1,50 & 2,40 & 13,03 & 17,63 & 414 & 6,56 & 83 & 0,56 & 0,15 & 0 & 0,73 & 2,12 & 0,33 & 1,92 \\
\hline K4 & 2.00 & 1,96 & 7,69 & 11,30 & 272 & 6,05 & 33 & 0,74 & 0,14 & 0, & 0,33 & 1,30 & 0,23 & 3,03 \\
\hline K8 & 1,10 & 2,73 & 10,72 & 14,25 & 318 & 6,07 & 32 & 0,50 & 0,12 & 0,6 & 0,34 & 1,14 & 0,24 & 2,33 \\
\hline Moyenne & 2,12 & 1,88 & 9,12 & 10,90 & 280 & 6,47 & 77 & 0,63 & 0,13 & 0,5 & 0,51 & 1,81 & 0,25 & 2,08 \\
\hline & 0,73 & 0,61 & 2,56 & 4,34 & 75 & 0,38 & 61 & 0,19 & 0,02 & 0,1 & 0,27 & 0,67 & 0,07 & 0,55 \\
\hline & 1.10 & 1,20 & 6.56 & 6.80 & 221 & 6.05 & 18 & 0.4 & 0,11 & 0,3 & 0,30 & 1,14 & 0,14 & 1,39 \\
\hline Maximum & 2,90 & 2.73 & 13,03 & 17,63 & 414 & 7,00 & 171 & 0,96 & 0,15 & 0,68 & 0,96 & 2,66 & 0,33 & 3.03 \\
\hline
\end{tabular}




\subsection{Comparaison et sélection des lacs selon le zooplancton}

Le tableau 4 présente les valeurs des biomasses de chacune des classes de taille et du zooplancton total dans chaque lac et en moyenne pour les lacs de coupe et les lacs de référence. Dans l'ensemble, les lacs à l'étude supportent des biomasses de zooplancton variant de 9,63 à $66,29 \mathrm{mg} \cdot \mathrm{m}^{-3}$. La biomasse totale de zooplancton et des classes de taille (53-100, 100-200, 200-500 et $>500 \mu \mathrm{m}$ ) est en moyenne plus importante dans les lacs de coupe (respectivement 43,$5 ; 6,6 ; 10,2 ; 9,6$ et $17,1 \mathrm{mg} \cdot \mathrm{m}^{-3}$ ) que dans les lacs de référence $\left(33,5 ; 5,5 ; 7,5 ; 6,7\right.$ et $\left.13,8 \mathrm{mg} \cdot \mathrm{m}^{-3}\right)$. Par contre, les classes de taille ont des importances relatives semblables pour les deux groupes de lacs. En moyenne, la classe de taille $>500 \mu \mathrm{m}$ représente environ $40 \%$ de la biomasse totale comparé à $16 \%$ pour la classe de taille 53-100 $\mu \mathrm{m}$. Les deux classes de taille intermédiaires, quant à elles, représentent $21 \%(200-500 \mu \mathrm{m})$ et $23 \%$ (100-200 $\mu \mathrm{m})$ de la biomasse du zooplancton total. Dans les lacs de référence, les biomasses de zooplancton ont une plus grande étendue pour la classe de taille $>500 \mu \mathrm{m}\left(1,4 \mathrm{à} 27,1 \mathrm{mg} \cdot \mathrm{m}^{-3}\right)$ et pour la biomasse totale $\left(9,6\right.$ à $\left.56,3 \mathrm{mg} \cdot \mathrm{m}^{-3}\right)$. Pour les lacs de coupe, les classes de taille $53-100 \mathrm{~mm}\left(7,3 \mathrm{à} 25,3 \mathrm{mg} \cdot \mathrm{m}^{-3}\right)$ et $100-200 \mu \mathrm{m}\left(4,1\right.$ à $\left.25,4 \mathrm{mg} \cdot \mathrm{m}^{-3}\right)$ ont une importante variation, mais c'est la biomasse totale qui a, encore une fois, la plus grande variation $\left(18,0\right.$ à $66,3 \mathrm{mg} \cdot \mathrm{m}^{-}$ 3). PATOINE et al. (2000) ont trouvé une biomasse moyenne de zooplancton total un peu plus élevée $\left(52,8 \mathrm{mg} \cdot \mathrm{m}^{-3}\right)$ pour 20 lacs de référence de la même région, comparativement à nos lacs. Dans cette étude comparative, les deux plus petites classes de taille $\left(53-100 \mu \mathrm{m}: 9,6 \mathrm{mg} \cdot \mathrm{m}^{-3} ; 100-200 \mu \mathrm{m}\right.$ : $10,5 \mathrm{mg} \cdot \mathrm{m}^{-3}$ ) avaient des biomasses moyennes semblables à celles décrites dans ce présent article, alors que la classe de taille $200-500 \mu \mathrm{m}$ avait une biomasse moyenne plus élevée en zooplancton $\left(18,1 \mathrm{mg} \cdot \mathrm{m}^{-3}\right)$ et la classe de taille $>500 \mu \mathrm{m}$ une biomasse moyenne un peu plus faible $\left(12,7 \mathrm{mg} \cdot \mathrm{m}^{-3}\right)$ que pour les lacs de référence retenus dans le cadre de notre étude.

La classe de taille de zooplancton $>500 \mu \mathrm{m}$ est la seule à sortir du cercle de contribution équilibrée $(r=0,7071)$ (figure $5 b$ ). Le premier axe explique $72,6 \%$ de la variation totale de la biomasse du zooplancton alors que le deuxième en explique $15,0 \%$. Selon la méthode du bâton brisé, le deuxième axe n'est pas significatif (valeur significative de $52,1 \%$ pour le premier axe et $27,1 \%$ (plus grand que $15,0 \%$ ) pour le deuxième axe). On remarque en effet que la distribution des lacs n'est pas très étendue sur le deuxième axe.

Le lac de référence $\mathrm{AB} 34$ a la plus petite valeur de biomasse pour la classe de taille $>500 \mu \mathrm{m}$, comparativement aux lacs de coupe distribués vers la gauche du graphique (figure $5 \mathrm{a}$ ). En général, les lacs de référence et de coupe ont des distributions réparties sur le premier axe, et aucun autre lac de référence n'a une distribution excentrique. Même si la position du lac AB34 diffère de celle des lacs de coupe pour la biomasse de zooplancton $>500 \mu \mathrm{m}$, ce lac sera retenu dans le groupe des lacs de référence car sa position se rapproche de celle du lac de coupe DF7 qui a aussi une petite biomasse pour la classe de taille $>500 \mu \mathrm{m}$. De plus, les deux autres ACPs sur la morphométrie et la qualité de l'eau montrent que le lac AB34 ressemble beaucoup plus aux lacs de coupe que les lacs AB35, AB40, K2 et N89. 
Tableau 4 Valeurs des biomasses du zooplancton total $\left(\mathrm{mg} \cdot \mathrm{m}^{-3}\right)$ et de chaque classe de taille dans chacun des lacs de coupe; moyenne, écarttype, minimum et maximum pour les dix lacs de référence et les six lacs de coupe. Classes de taille du zooplancton : 53-100 $\mu \mathrm{m}, 100-$ $200 \mu \mathrm{m} ; 200-500 \mu \mathrm{m},>500 \mu \mathrm{m}$. La biomasse du zooplancton total est la somme des biomasses de chacune des classes de taille.

Table 4 Values of total zooplankton biomass $\left(\mathrm{mg} \cdot \mathrm{m}^{-3}\right)$ and biomass in each size class in each cut lake; mean, standard deviation, minimum and maximum for the ten reference lakes and the six cut lakes. Zooplankton size classes: $53-100 \mu \mathrm{m}, 100-200 \mu \mathrm{m}, 200-500 \mu \mathrm{m},>500 \mu \mathrm{m}$. Total zooplankton biomass is the sum of the biomasses in each size class.

\begin{tabular}{cccccc}
\hline $\begin{array}{c}\text { Lacs } \\
\text { de référence }\end{array}$ & $\begin{array}{c}\mathbf{5 3 - 1 0 0} \\
(\boldsymbol{\mu m})\end{array}$ & $\begin{array}{c}\mathbf{1 0 0 - 2 0 0} \\
(\boldsymbol{\mu m})\end{array}$ & $\begin{array}{c}\mathbf{2 0 0 - 5 0 0} \\
(\boldsymbol{\mu m})\end{array}$ & $\begin{array}{c}>\mathbf{5 0 0} \\
(\boldsymbol{\mu m})\end{array}$ & $\begin{array}{c}\text { Zooplankton } \\
\text { Total }\end{array}$ \\
\hline AB34 & 1,76 & 3,91 & 2,61 & 1,35 & 9,63 \\
AB35 & 2,58 & 3,96 & 2,97 & 4,84 & 14,34 \\
AB40 & 8,24 & $\mathbf{1 1 , 6 0}$ & 8,50 & 8,85 & 37,18 \\
CSL5 & 4,16 & 2,86 & 1,82 & 15,49 & 24,33 \\
DA4 & 8,80 & 14,49 & 16,37 & 11,31 & 50,97 \\
DF4 & 8,80 & 10,61 & 9,82 & 27,10 & 56,32 \\
K2 & 8,44 & 11,15 & 7,15 & 25,75 & 52,49 \\
N35 & 6,98 & 6,30 & 7,47 & 21,23 & 41,99 \\
N43 & 3,38 & 5,74 & 6,83 & 10,94 & 26,90 \\
N89 & 2,04 & 4,29 & 3,66 & 11,15 & 21,15 \\
Moyenne & 5,52 & 7,49 & 6,72 & 13,80 & 33,53 \\
Écart-type & 3,00 & 4,09 & 4,36 & 8,56 & 16,63 \\
Minimum & 1,76 & 2,86 & 1,82 & 1,35 & 9,63 \\
Maximum & 8,80 & 14,49 & 16,37 & 27,10 & 56,32 \\
\hline Lacs & $\mathbf{5 3 - 1 0 0}$ & $\mathbf{1 0 0 - 2 0 0}$ & $\mathbf{2 0 0 - 5 0 0}$ & $>\mathbf{5 0 0}$ & Zooplankton \\
de référence & $(\boldsymbol{\mu m})$ & $\mathbf{( \mu m )}$ & $(\boldsymbol{\mu m})$ & $\mathbf{( \mu m )}$ & Total \\
\hline DA2 & 7,94 & 13,18 & 25,41 & 19,76 & 66,29 \\
DF2 & 6,51 & 11,06 & 9,64 & 17,81 & 45,02 \\
DF7 & 1,85 & 4,75 & 4,11 & 7,25 & 17,97 \\
DF9 & 4,79 & 6,52 & 4,52 & 25,26 & 41,09 \\
K4 & 8,66 & 11,15 & 5,47 & 15,96 & 41,23 \\
K8 & 10,02 & 14,77 & 8,30 & 16,34 & 49,43 \\
Moyenne & 6,63 & 10,24 & 9,58 & 17,06 & 43,51 \\
Écart-type & 2,95 & 3,86 & 8,06 & 5,88 & 15,62 \\
Minimum & 1,85 & 4,75 & 4,11 & 7,25 & 17,97 \\
Maximum & 10,02 & 14,77 & 25,41 & 25,26 & 66,29 \\
\hline
\end{tabular}

\subsection{Sélection finale des lacs de référence}

D'après les trois ordinations des 10 lacs de référence et des 6 lacs de coupe basées sur la morphométrie, la qualité de l'eau et la biomasse de zooplancton, 4 lacs (AB35, N89, AB40,K2) ont été éliminés lors de la sélection du groupe de lacs de référence les plus similaires possible aux lacs de coupe. Le lac K2 a été éliminé sur la base de valeurs extrêmes pour l'aire et le volume du lac (figure 3), le lac N89 pour une transparence au Secchi plus élevée et des concentrations en COD, NT et PT moins élevées que celles des lacs de coupe 
(figure 4), le lac AB40 parce qu'il a de faibles valeurs de $\mathrm{pH}$, d'alcalinité, de $\mathrm{Mg}^{2+}$ et de $\mathrm{Ca}^{2+}$ (figure 4) et le lac AB35 pour ses valeurs plus élevées pour la profondeur maximale et le volume du lac d'une part (figure 3 ), et pour la plus forte transparence au Secchi et des concentrations plus faibles en COD, NT et PT d'autre part (figure 4).

En définitive, les six lacs sélectionnés pour le groupe de référence sont les lacs AB34, CSL5, DA4, DF4, N35 et N43. Nous avons donné la priorité aux variables morphométriques pour la sélection des lacs de référence car ces variables ont le plus d'effet sur le niveau de ruissellement et d'apports en éléments nutritifs lors de fortes précipitations ; elles peuvent donc amplifier l'impact des coupes forestières. Ainsi, si les lacs de référence ont en moyenne une aire de drainage plus élevée que les lacs de coupe, une forte précipitation après la perturbation favorisera un apport accru en nutriments dans les lacs de référence, ce qui pourrait avoir un effet ascendant (bottom-up) sur la communauté de zooplancton. Par contre, dans les lacs de coupe dont l'aire de drainage serait plus faible, cette forte précipitation aura un effet moindre. Ces différences au niveau de la morphométrie pourraient sous-estimer la valeur du delta ( $\Delta$ : différence entre les lacs de référence et les lacs de coupe) après la perturbation (2001-2002) et diminuer les chances de détecter des effets significatifs de la perturbation.

\subsection{Validation de la sélection des lacs de référence}

L'hypothèse nulle selon laquelle les lacs de référence sélectionnés ont des caractéristiques semblables aux lacs de coupe n'est pas rejetée par les trois analyses de redondance (RDA) sur les variables de la morphométrie $(P=$ $0,1023)$, de la qualité de l'eau $(P=0,9409)$ et du zooplancton $(P=0,4803)$. Nos deux groupes de lacs ne diffèrent donc pas quant à ces trois types de descripteurs et devraient avoir des réponses similaires vis-à-vis des variations naturelles dans leur environnement. Ces résultats soutiennent la sélection des lacs de référence faite à partir de la lecture des graphiques d'ordination des analyses en composantes principales sur les trois types de descripteurs.

L'approche $\mathrm{BACl}$ sera donc appliquée aux deux groupes de lacs suivants:

Lacs de coupe : $\quad$ DA2, DF2, DF7, DF9, K4 et K8

Lacs de référence : AB34, CSL5, DA4, DF4, N35 et N43

L'analyse de redondance (RDA), une méthode multivariée, a certains avantages sur les méthodes d'analyses statistiques univariées. Elle permet de vérifier si les deux groupes de lacs sont différents quant à plusieurs variables et non par rapport à une seule variable comme c'est le cas pour les ANOVA ou le test non paramétrique de Kruskal-Wallis (LEGENDRE et LEGENDRE, 1998).

\section{$4-$ CONCLUSION GÉNÉRALE}

La méthode de sélection de sites de référence développée ici pour l'application d'une approche $\mathrm{BACl}$ visant à évaluer l'impact d'une coupe forestière 
sur la biomasse de zooplancton dans les lacs de la forêt boréale, peut être généralisée pour toute étude d'impact d'une perturbation sur une ou plusieurs composantes d'un écosystème. Dans le cadre de notre étude, cette méthode permet d'éliminer les lacs ayant une valeur extrême, pour un ou plusieurs descripteurs de la morphométrie, de la qualité des eaux, et de la biomasse du zooplancton, grâce à des ordinations produites par des analyses en composantes principales (ACP) basées sur chaque type de descripteurs. Seuls les lacs présentant les plus fortes similarités vis-à-vis des lacs de coupe pour chacun de ces descripteurs ont été retenus dans le groupe de référence.

Bien que ce choix fasse appel à un certain jugement et à des compromis afin de maintenir deux ensembles de lacs équilibrés (six lacs de référence, six lacs de coupe), les analyses de redondance (RDA) ont montré que la sélection effectuée permettait de comparer, dans le cadre d'une approche $\mathrm{BACl}$, deux groupes de lacs ayant les mêmes caractéristiques limnologiques avant la perturbation du bassin versant par les coupes forestières.

Puisque les lacs de référence sélectionnés et les lacs de coupe ne sont pas différents avant la perturbation, l'approche $\mathrm{BACl}$ nous permettra de tester si les coupes forestières ont un impact sur la biomasse du zooplancton dans les lacs de la forêt boréale, en mesurant si les différences observées avant et après les coupes forestières dans la biomasse de zooplancton de chacun des lacs de coupe, telle que déterminée à partir de la mesure des biomasses de quatre classes de taille du zooplancton, sont significatives comparativement à la variation naturelle moyenne dans les lacs de référence.

\section{REMERCIEMENTS}

Cette étude a été réalisée dans le cadre d'un projet du Réseau de centre d'excellence sur la gestion durable des forêts (RCE-GDF) par une équipe de chercheurs du GRIL (Groupe de recherche interuniversitaire en limnologie et en environnement aquatique) de l'Université de Montréal. La recherche a été financée par le Conseil de recherche en sciences naturelles et en génie (CRSNG) en partenariat avec l'industrie forestière du Canada (Abitibi-Consolidated, Cartons Saint-Laurent, Donohue, Kruger) et le fonds pour la formation de chercheurs et l'aide à la recherche du Québec (FCAR). Nous remercions Mathieu BONDALLAZ, M.Sc. pour sa participation à l'échantillonnage et à l'analyse du zooplancton en 2000 et 2001 , de même que pour ses précieux conseils sur la logistique des campagnes d'échantillonnage et les techniques en laboratoire, Pierre LEGENDRE et Ginette MÉTHOT pour l'aide lors des analyses statistiques, Richard CARIGNAN et Dolors PLANAS pour les données de morphométrie et de qualité d'eau. Nous voulons également souligner la collaboration de Pierre D'ARCY et Marc BÉLANGER, responsables de la logistique de terrain, ainsi que des autres étudiants gradués, des techniciens et des pilotes d'avion. Cet article fait suite à une communication par affiche présentée par Lyne DUHAIME et Bernadette PINEL-ALLOUL au congrès CILEF-2003 tenu du 27 juillet au $1^{\mathrm{er}}$ août à Montréal (Québec, Canada). 


\section{RÉFÉRENCES BIBLIOGRAPHIQUES}

BAYLEY S. E., SCHINDLER D. W., BEATY K. G., PARKER B. R., STAINTON M. P., 1992. Effects of multiple fires on nutrients yields from streams draining boreal forest and fen watersheds. Can. J. Fish. Aquat. Sci., 49, 577-583.

BERNSTEIN B. B., ZALINSKI J., 1983. An optimum sampling design and power tests for environmental biologists. J. Environ. Manage, 16, 35-43.

BONDALLAZ M., 2002. Étude expérimentale de l'impact de la coupe forestière sur la biomasse et la structure du zooplancton des lacs de l'écozone boréale. Thèse de Maîtrise, Dépt. Sciences biologiques, Université de Montréal. $85 \mathrm{p}$.

BOX G. E. P., TIAO G. C., 1975. Intervention analysis with application to economic and environmental problems. J. Amer. Statist. Ass., 70, 70-79.

CARIGNAN R., D'ARCY P., LAMONTAGNE S., 2000. Comparative impacts of fire and forest harvesting on water quality in Boreal Shield lakes. Can. J. Fish. Aquat. Sci., 57 (suppl. 2), 105-117.

CURRIE D. J., DILWORTH-CHRISTIE P., CHAPLEAU F., 1999. Assessing the strength of top-down influences on plankton abundance in unmanipulated lakes. Can. J. Fish. Aquat. Sci., 56, 427436.

D'ARCY P., CARIGNAN R., 1997. Influence of watersheds topography on waters quality in southestern Québec Shield lakes. Can. J. Fish. Aquat. Sci., 54, 2215-2227.

DEL GIORGIO P. A., COLE J. J., CIMBLERIS A., 1997. Respiration rates in bacteria exceed phytoplankton production in unproductive aquatic systems. Nature, 385, 148-151.

DEL GIORGIO P. A., PETERS R. H., 1994. Patterns in the planktonic $P$ : $R$ ratios in lakes: influence of lake trophy and dissolved organic carbon. Limnol. Oceanogr., 39: 772-787.

DILLON P. J., RIGLER F. H., 1974. The phosphorus-chlorophyll relationship in lakes. Limnol. Oceanogr., 19, 767-773.

FILION J.-M., CHAIN P., FUTTER M., 1993. Cantilevering vertical tow nets to reduce tow-line-induced zooplankton avoidance. J. Plankton Res., 15, 581-587.

GARCIA E., CARIGNAN R., 1999. Impact of wildfire and clear-cutting in the boreal forest on methyl mercury in zooplankton. Can. J. Fish. Aquat. Sci., 56, 339-345.

GREEN R. H., 1979. Sampling design and statistical methods for environmental biologist. Wiley Interscience, New York.

HOULE D., CARIGNAN R., LACHANCE M., DUPONT J., 1995. Dissolved organic carbon and sulfur in southwestern Québec lakes: Relationships with catchment and lake properties. Limnol. Oceanogr., 40, 710-717.

JONES R. I., 1992. The influence of humic substances on lacustrine planktonic food chains. Hydrobiologia, 229, 73-91.

LAMONTAGNE S., CARIGNAN R., D'ARCY P., PRAIRIE Y., PARÉ D., 2000. Element export in runoff from eastern Canadian Boreal Shield drainage basins following forest harvesting and wildfires. Can. $J$. Fish. Aquat. Sci., 57 (suppl. 2), 118-128.

LEGENDRE P., LEGENDRE L., 1998. Numerical Ecology, $2^{\text {nd }}$ English edition. Elsevier Science BV, Amsterdam.

LEHMANN R., 1994. Forest clearance and lake water quality on the Canadian Shield. M.Sc. McGill Univ. 73 p.

MCQUEEN D. J., POST J. R., MILLS E. L., 1986. Trophic relationships in freshwater pelagic ecosystems. Can. J. Fish. Aquat. Sci., 43, 1571-1581.

OSENBERG C. W., SCHMITT R. J., 1996. Detecting ecological impacts caused by human activities. In "Detecting ecological impacts, concepts and applications in coastal habitats". SCHMITT, R. J. et C. W. OSENBERG [Ed.] pp. 3-16.

PACE M. L., 1984. Zooplankton community structure, but not biomass, influences the phosphorus-chlorophyll a relationship. Can. J. Fish. Aquat. Sci., 41, 1089-1096.

PATOINE A., PINEL-ALLOUL B., PREPAS E. E., 2002a. Effects of catchment perturbations by logging and wildfires on zooplankton species richness and composition in Boreal Shield lakes. Freshwater Biol. , 47, 1996-2014. 
PATOINE A., PINEL-ALLOUL B., PREPAS E. E., 2002b. Influence of catchment deforestation by logging and natural forest fires on crustacean community size structure in lakes of the Eastern Boreal Canadian forest. J. Plankton Res., 24, 601-616.

PATOINE A., PINEL-ALLOUL B., PREPAS E. E., CARIGNAN R., 2000. Do logging and forest fires influence zooplankton biomass in Canadian boreal shield lakes? Can. J. Fish. Aquat. Sci., 57 (Suppl. 2), 155-164.

PREPAS E. E., PINEL-ALLOUL B., PLANAS D., MÉTHOT G., PAQUET S., REEDYK S., 2001. Forest harvest impacts on water quality and aquatic biota on the Boreal Plain: introduction to the TROLS lake program. Can. J. Fish. Aquat. Sci., 58, 421-436.

RASK M., NYBERG K., MARKKANEN S.-L., OJALA A., 1998. Forestry in catchments: effect on water quality, plankton, zoobenthos and fish in small lakes. Boreal Environ. Res., 3, 75-86.

SAKAMOTO M., 1966. Primary production by phytoplankton community in some Japanese lakes and its dependence on lake depth. Arch. Hydrobiol., 62, 1-28.

SCHINDLER D. W., BAYLEY S. E., PARKER B. R., BEATY K. G., CRUIKSHANK D. R., FEE E. J., SCHINDLER E. U., STAINTON M.P., 1996. The effects of climatic warming on the properties of boreal lakes and streams at the Experimental Lake Area, Northwestern Ontario. Limnol. Oceanogr., 41, 1004-1017.
SCHINDLER D. W., BAYLEY S. E., CURTIS P. J., PARKER B. R., STAINTON M. P., KELLY C. A., 1992. Natural and mancaused factors affecting the abundance and cycling of dissolved organic substances in Precambrian shield lakes. Hydrobiologia, 229, 1-21.

SCHINDLER D. W., 1978. Factors regulating phytoplankton production and standing crop in the world's freshwaters. Limnol. Oceanogr., 23, 478-486.

STEEDMAN R. J., 2000. Effects of experimental clearcut logging on water quality in three small boreal forest lake trout (Salvelinus namaycush) lakes. Can. J. Fish. Aquat. Sci., 57 (Suppl. 2), 92-96.

STEEDMAN R. J., KUSHNERIUK R. S., 2000. Effects of experimental clearcut logging on thermal stratification, dissolved oxygen, and lake trout (Salvelinus namaycush) habitat volume in three small boreal forest lakes. Can. J. Fish. Aquat. Sci., 57 (Suppl. 2), 82-91.

STEWART-OATEN A., BENCE J. R., 2001. Temporal and spatial variation in environmental impact assessment. Écol. Monogr., 71, 305-339.

STEWART-OATEN A., MURDOCH W. W., PARKER K. R., 1986. Environmental impact assessment: "pseudoreplication" in time? Ecology, 67, 929-940.

TER BRAAK C. J. F., SMILAUER P., 1998. CANOCO Reference Manual and User's Guide to Canoco for Windows: Software for Canonical Community Ordination (version 4). Microcomputer Power, Ithaca, New York. 